\title{
Direct observation of defect modes in molecular aggregate analogs
}

\author{
M. Baraclough $\odot,{ }^{*}$ I. R. Hooper $\odot$, and W. L. Barnes ${ }^{\dagger}$ \\ Department of Physics and Astronomy, University of Exeter, Stocker Road, Exeter EX4 4QL, United Kingdom
}

(Received 17 June 2020; accepted 25 November 2020; published 18 December 2020)

\begin{abstract}
In this work we investigate defect modes localized at the ends and within the bulk of $1 \mathrm{D}$ metamaterial analogs of molecular aggregates. The study is undertaken in the microwave regime, where the cm scale of the metamaterial analog provides an opportunity to directly probe the modes deep within their near fields in ways not easily achieved in molecular systems. To demonstrate the power of this approach we compare our observations to predictions from a simple $\mathrm{Su}$, Schrieffer, and Heeger (SSH) model and find good qualitative agreement.
\end{abstract}

DOI: 10.1103/PhysRevB.102.224105

\section{INTRODUCTION}

Molecular aggregates have long been of interest to scientists [1] and continue to be a lively and fruitful topic of research [2,3]. Studies of aggregate phenomena have led to advances in the field of sensing [4,5], as well as offering a more fundamental understanding of natural light-harvesting processes such as those found in the photosynthetic structures of bacteria [6,7]. Many of the properties of molecular aggregates arise due to nanometer scale near-field interactions between the constituent molecules. However, experimental investigations are typically constrained to probing the far-field consequences of these near-field interactions [8], and even those techniques that are able to probe the near-field are not able to do so on the intermolecular scale [9]. As a result the disorder in these systems, which is known to play an important role in, for example, exciton transport [10], can only be inferred rather than directly probed.

In previous work we showed [11] how some of the properties of molecular aggregates can be replicated at the $\mathrm{cm}$ scale through the use of metamaterial analogs. Metamaterials, artificial materials whose properties arise from their structure rather than from the inherent properties of their constituent materials, are usually employed to provide material properties that cannot be found in nature [12]. However, they are also well suited to enabling phenomena usually found only in very small-scale systems to be replicated in analogous longer scale systems. This longer available length scale leads to one of the strengths of the metamaterial approach; it provides a degree of structural control that would be very hard to realize at smaller, molecular, length scales. In turn, this extra control allows physical processes to be probed in ways that would not otherwise have been possible. Molecular aggregates are a good example of this; an electromagnetic metamaterial analog is well suited to replicating their subwavelength nature and the near-field interactions that dominate their behavior [13]. Another powerful recent example of using $\mathrm{cm}$-scale structures

\footnotetext{
*mb504@exeter.ac.uk

†w.1.barnes@exeter.ac.uk
}

and RF techniques to probe molecular analogs is the work of Rustomji et al. to look at dipole-dipole interactions [14].

In this work we make use of the metamaterial approach to mimic a molecular aggregate. We create a linear chain of metamolecule resonators and, by structurally altering the order of this chain, we introduce defects that support localized modes. We subsequently directly probe the near fields of these defect modes by employing electrically-small nearfield antennas and compare our results to those predicted by the Su-Schrieffer-Heeger (SSH) model. For all of its wide applicability the SSH model was originally used to map the probability density of electrons along chains of polyacetylene and to predict the effect of disorder in the properties of these chains [15]. By relating the defect types presented here to those predicted by the SSH model we further validate the power and value of the metamaterial approach to studying molecular aggregate properties.

\section{THE SSH MODEL}

$\mathrm{Su}$, Schrieffer, and Heeger introduced a very effective toy model for a molecular aggregate that employs a tight-binding Hamiltonian to model the energies and field intensities in a system of resonators that are coupled to each other with bonds of alternating strength $[15,16]$, see Fig. 1(a). The SSH model was originally conceived in order to understand the modes supported by trans-polyacetylene, where carbon atoms are coupled via alternating single and double bonds. With this model the existence of dispersionless soliton (solitary wave) states was predicted, states arising from phase changes due to the periodic carbon structure that could explain some of the substance's unique properties. In 2016 Meier et al. directly observed the soliton in polyacetylene that had first been predicted in 1979 [17]. The form of the SSH model used in this paper, along with how its solutions lead to localized states, is described below.

The Hamiltonian in Eq. (1) represents a system of eight resonators, each of which has a resonance frequency $\omega$, the resonators are coupled via two alternating nearest neighbor coupling terms of differing strength. This arrangement results in four 'cells,' with $k_{A}$ being the coupling between two 
(a)

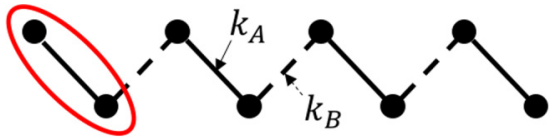

(b)

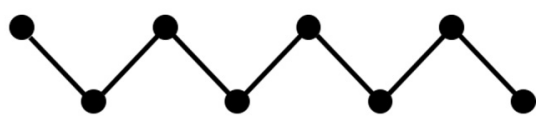

(c)

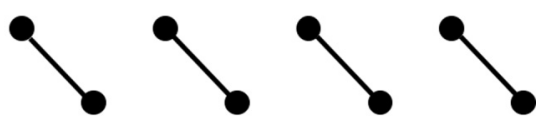

(d)
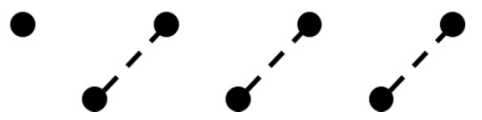

FIG. 1. SSH structure. (a) A schematic of a chain of eight resonators, each with a resonance frequency $\omega$. Two resonators within a 'cell' are coupled together via an intracell coupling term $k_{A}$ (red ellipse), with each cell in the chain being coupled to neighboring cells via a second intercell coupling term $k_{B}$. When $k_{A} \neq k_{B}$ the system deviates from the monoatomic, single banded, case (b) and a band gap will emerge in the dispersion of the chain. (c) In the dimer limit when $k_{B}=0$ and $k_{A} \neq 0$ the chain consists of four isolated dimers. Each dimer will support two modes with frequencies that will lie above and below $\omega$. The difference in frequency between them is determined by the magnitude of $k_{A}$. (d) In the other dimer limit, when $k_{A}=0$ and $k_{B} \neq 0$, there are three isolated dimers, and in this case the difference in frequency between their modes is determined by $k_{B}$. In addition, there are two isolated resonators at each end of the chain. These isolated resonators will have resonance frequencies of $\omega$, which lie between those of the dimers, i.e., within the band gap. It is not just in the dimer limit that these 'edge' modes appear, rather it is only necessary that $k_{A}<k_{B}$.

resonators within the same cell (intracell coupling) and $k_{B}$ being the coupling between cells (intercell coupling).

$$
\hat{H}=\left[\begin{array}{cccccccc}
\omega & k_{A} & 0 & 0 & 0 & 0 & 0 & 0 \\
k_{A} & \omega & k_{B} & 0 & 0 & 0 & 0 & 0 \\
0 & k_{B} & \omega & k_{A} & 0 & 0 & 0 & 0 \\
0 & 0 & k_{A} & \omega & k_{B} & 0 & 0 & 0 \\
0 & 0 & 0 & k_{B} & \omega & k_{A} & 0 & 0 \\
0 & 0 & 0 & 0 & k_{A} & \omega & k_{B} & 0 \\
0 & 0 & 0 & 0 & 0 & k_{B} & \omega & k_{A} \\
0 & 0 & 0 & 0 & 0 & 0 & k_{A} & \omega
\end{array}\right]
$$

This Hamiltonian can be solved to obtain its eigenvalues, which correspond to the frequencies of the resonances supported by the chain of resonators, and its eigenvectors, which correspond to the field amplitude distribution along the chain for each of the eigenvalues. We will consider two cases: (1) where $k_{A}>k_{B}$ and (2) where $k_{B}>k_{A}$. The case of $k_{A}=k_{B}$ is trivial and corresponds to that of a monoatomic chain and is not of interest here [see Fig. 1(b)].

To gain some physical insight we begin by considering the limits of $k_{A}>k_{B}=0$ and $k_{B}>k_{A}=0$, these are the so-called 'dimer limits' where the chain consists of a series of noninteracting dimers. In the $k_{A}>k_{B}=0$ dimer limit we
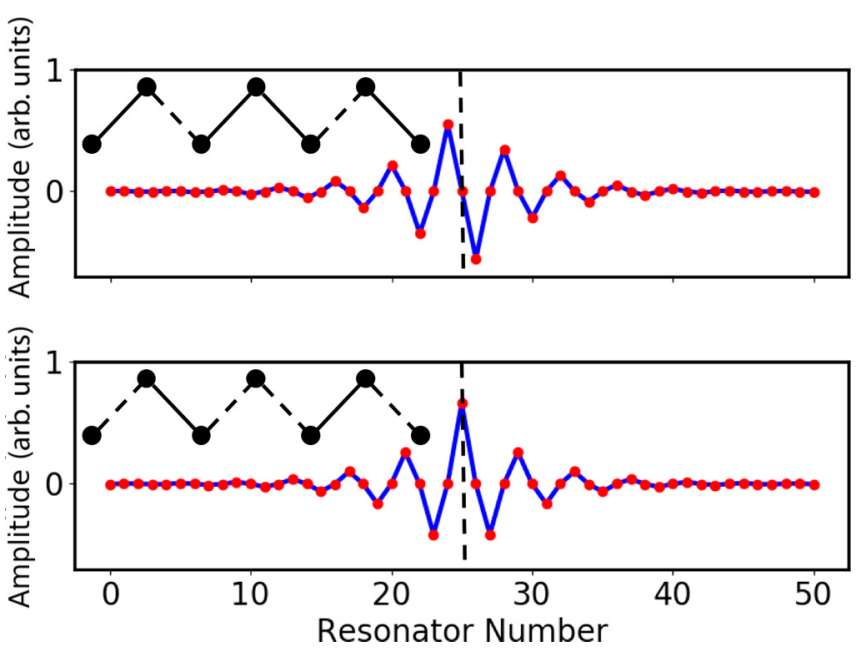

FIG. 2. Boundary mode eigenvectors. Top: Calculated eigenvector of a fifty resonator long chain in which the relative strengths of $k_{A}$ and $k_{B}$ are switched midway along the chain such that the resonator at the boundary is strongly coupled to both its nearest neighbors. This boundary mode has an asymmetric form. Bottom: The same as the top figure, but in this case the position of the switch occurs such that the resonator at the boundary is weakly coupled to its neighbors. In this case the boundary mode is symmetric. Boundaries in both cases are marked by the vertical black dashed line.

find just two resonance frequencies, the higher and lower energy modes of each of the four dimers in our chain, see Fig. 1(c), with the difference in energy between them being determined by the strength of the intracell coupling. The case when $k_{B}>k_{A}=0$ is a little more interesting. Here we find three dimers within the body of the chain, but in addition we now have two independent resonators at the ends of the chain [see Fig. 1(d)]. These independent resonators will have a resonance frequency of $\omega$, which will lie between the resonance frequencies of the dimers, i.e., within the band gap. If we move away from the dimer limit by increasing the intracell coupling, $k_{A}$, the localization of the dimer modes is removed, resulting in a discrete set of modes that are supported by the entire chain. However, the localized modes at the terminations remain within the band gap_-all that changes is the degree of localization, which decreases as the coupling terms approach parity, along with the size of the band gap.

It is not just the terminations of the chain that can support localized modes, defects can also be introduced into the bulk of the chain. Consider a case where, partway along a chain, we switch the relative magnitudes of $k_{A}$ and $k_{B}$ such that we have $k_{A}>k_{B}$ on one side of a resonator and $k_{B}>k_{A}$ on the other. Depending upon whether we perform this switch at the site of a $k_{A}$ or a $k_{B}$ we get two distinct cases, one where the resonator at the boundary is strongly coupled to both its nearest neighbors, and one where it is weakly coupled to its nearest neighbors, see the insets of Fig. 2. If we calculate the eigenvalues for both cases we can identify localized modes centered at the boundaries where the switch occurs, which once again have frequencies that occur within the band gap. However, the eigenvectors for the two cases show distinctly different behaviors, as shown in Fig. 2. In the case where the resonator at the boundary is strongly coupled to both its 


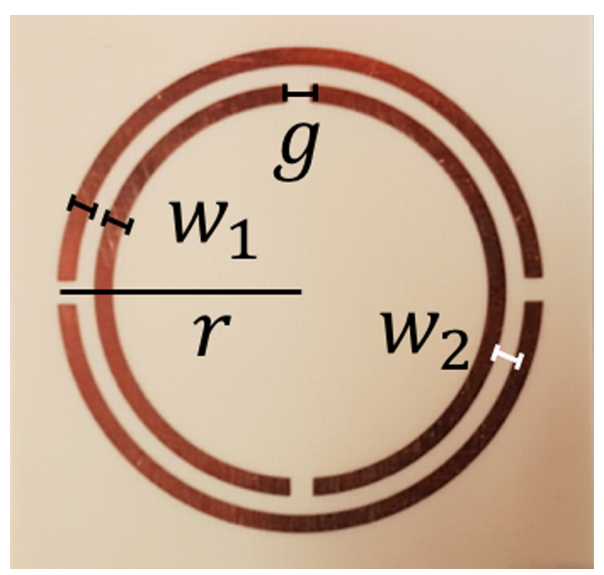

FIG. 3. The metamolecule. The metamolecules used in this study are based on the split-ring resonator, a loop of wire with a split that acts as a resonant circuit. Dark regions correspond to copper-coated areas of a dielectric substrate, light regions correspond to the bare substrate. The structure comprises two double split rings so as to remove any bianisotropy. $r=16 \mathrm{~mm}, w_{1}=w_{2}=1.2 \mathrm{~mm}$, and $g=$ $1.5 \mathrm{~mm}$.

nearest neighbors, that resonator will have zero amplitude (we will call this the 'asymmetric' boundary case based on the shape of the field amplitudes), whereas when the resonator at the boundary is weakly coupled to its neighbors it has maximum amplitude (the 'symmetric' boundary case). These two cases can be directly related to the soliton and antisoliton states of polyacetylene [18].

From this examination of the SSH model, we have identified three distinct localized modes that could be supported by our aggregatelike metamaterial chains: An 'edge' mode when the coupling between resonators in a cell is weaker than the coupling between cells and two boundary modes in the bulk with different forms that depend upon the nature of the boundary. In the following we discuss the designs of our analogs and report measurements that directly probe the forms of these three types of localized modes.

\section{METAMOLECULE DESIGN}

The metamolecule design used in this study, pictured in Fig. 3, has its basis in the split-ring resonator (SRR). However, the standard SRR, popularized by Pendry et al. [19], is bianisotropic, with coupled electric and magnetic dipole moments. This would be an undesirable feature for our metamolecules since the constituent molecules in a real aggregate will possess only electric dipole moments, if our analog has two different dipole coupling terms this will significantly complicate matters [20,21]. To overcome this, our design makes use of four splits arranged symmetrically so that, for the lowest frequency eigenmode of an individual resonator, the net electric dipole moment is zero. This leaves only a magnetic dipole moment, aligned through the center of the ring, which dominates any coupling between neighboring metamolecules. Note that whether the coupling between the resonators is via electric or magnetic dipole moments does not alter the underlying physics.

The metamolecules were fabricated using standard printed circuit board processing, using $35 \mu \mathrm{m}$ thick copper on Rogers
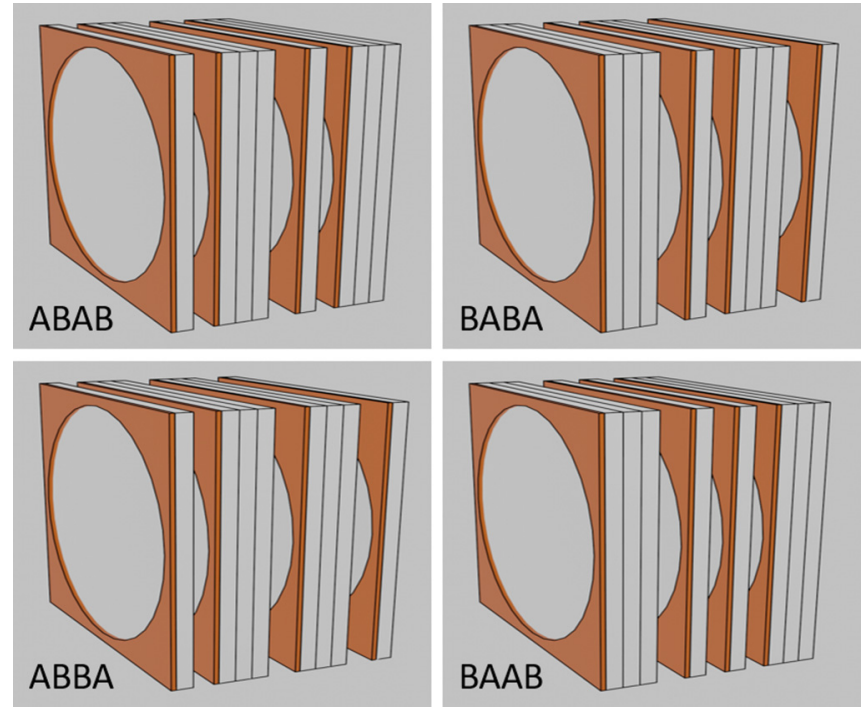

FIG. 4. Chain formations. Exploded schematics of the four chain formations where A refers to copper (orange) and a dielectric spacer (white) while B refers to copper and three dielectric spacers. The top row shows two units cells each of the ABAB (left) and BABA (right) formations. The bottom row shows the formations of the two defect structures going from $\mathrm{AB}$ to BA periodicity (left) and from BA to $\mathrm{AB}$ periodicity (right).

4350B material $\left(\epsilon_{r}=3.48\right)$ with a thickness of $1.55 \mathrm{~mm}$. The lowest order resonance frequency of a single resonator symmetrically bounded by the substrate material was $1.40 \mathrm{GHz}$.

To form our chains we stacked our resonators axially, while altering the inter- and intracell coupling strengths by inserting additional blank substrates (without any copper layer) in appropriate positions. Different chains were formed for each of the following four experiments: two chains to investigate edge modes with $k_{A}>k_{B}$ and $k_{A}<k_{B}$, and two chains to investigate symmetric and asymmetric defect (boundary) modes when subchains with switched values of $k_{A}$ and $k_{B}$ are joined together. Each chain consisted of 94 resonators. See Fig. 4 for details of the formations of each chain.

\section{MEASUREMENTS AND DISCUSSION}

All measurements were conducted using an Anritsu Vectorstar MS4644A Vector Network Analyser (VNA). To excite any modes supported by our chains with $k_{B}>k_{A}$ and $k_{B}<k_{A}$ a small loop antenna, resonant far from the the spectral range of interest, was placed at one end of the chain adjacent to one of the splits in the rings. The near fields of the excited modes were then probed using a second loop antenna that was swept along the length of the sample while measuring the transmission (S21) between the exciting and probing antennas, see the top of Fig. 5. The dimensions of the antennas were such that, at the frequencies studied here, they were far from resonance and minimally perturbed the modes of the chains. The magnitude of the power transmission between the antennas was measured as a function of position along the chains over the frequency range 1.2 to $1.8 \mathrm{GHz}$.

In Fig. 6(a) we show the transmission data for the chain with $k_{A}>k_{B}$. Note that the transmission is shown for only the 


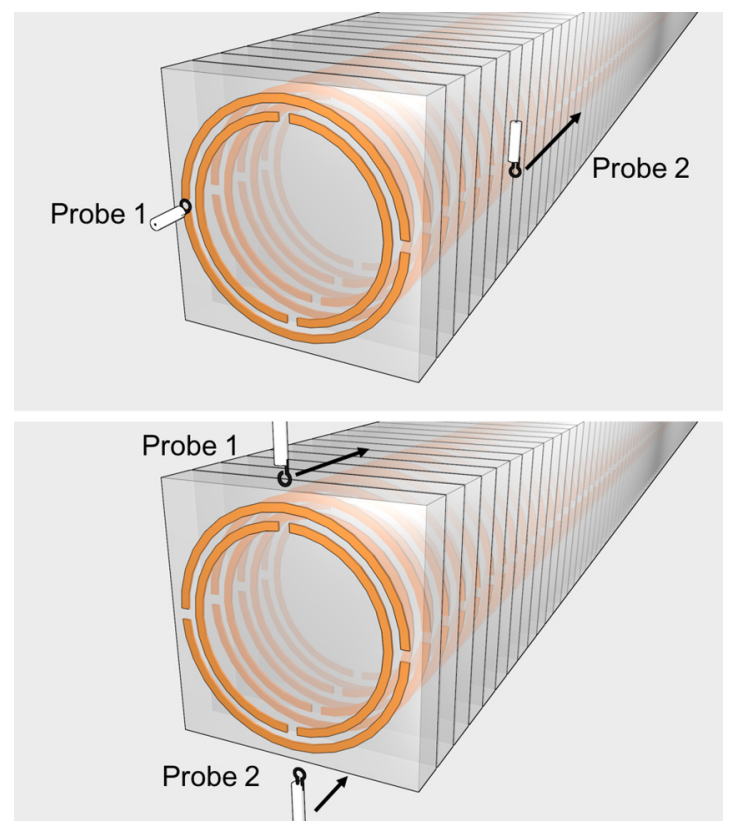

FIG. 5. Scanning methods. A schematic of the two methods used to detect localized modes within a chain of resonators. In both probe 1 is the exciting antenna and probe 2 is the receiving antenna. Black arrows indicate which probes are scanned as part of the measurement. (Top) The scanning method for probing edge modes uses a loop antenna (probe 1) placed at a split on the outer ring of the first resonant element so as to excite the modes of the chain. The loop was orientated in the plane of the resonator. A second loop antenna (probe 2) was scanned along the edge of the chain with the same loop orientation. The transmission between the two antennas was measured. (Bottom) The scanning method for probing localized modes within the bulk of the chain. Two loop antennas (probes 1 and 2) are scanned in tandem along opposite edges of the chain and the transmission between them measured.

first $60 \mathrm{~mm}$ of the $290 \mathrm{~mm}$ long chain. At low frequencies the chain supports collective modes that are distributed along the entire length of the chain. These appear as long-range oscillations in the transmission as a function of distance. As the frequency is increased the wavelength of these collective modes becomes shorter until a band gap is encountered at approximately $1.4 \mathrm{GHz}$. This band gap extends to $1.65 \mathrm{GHz}$, beyond which oscillatory modes become evident once again. Within the band gap there is some direct transmission between the two antennas but no transmission mediated via modes of the chain. No localized mode at the end of the chain within the band gap is observed as expected when $k_{A}>k_{B}$.

In Fig. 6(b) we show equivalent data for the case where $k_{B}>k_{A}$. The data look very similar to those in Fig. 6(b), except that in this case there is a clear mode within the band gap, at $1.52 \mathrm{GHz}$ that decays into the chain, as predicted by the SSH model. The mode's rate of decay is governed by multiple factors but primarily the spectral proximity of the localized mode to either of the associated band gap edges. This can be tuned by altering the resonance frequency of the end resonator or by decreasing the difference between $k_{A}$ and $k_{B}$ and partially closing the band gap.

To probe the boundary modes, we need to alter our experimental setup somewhat since any mode localized in the

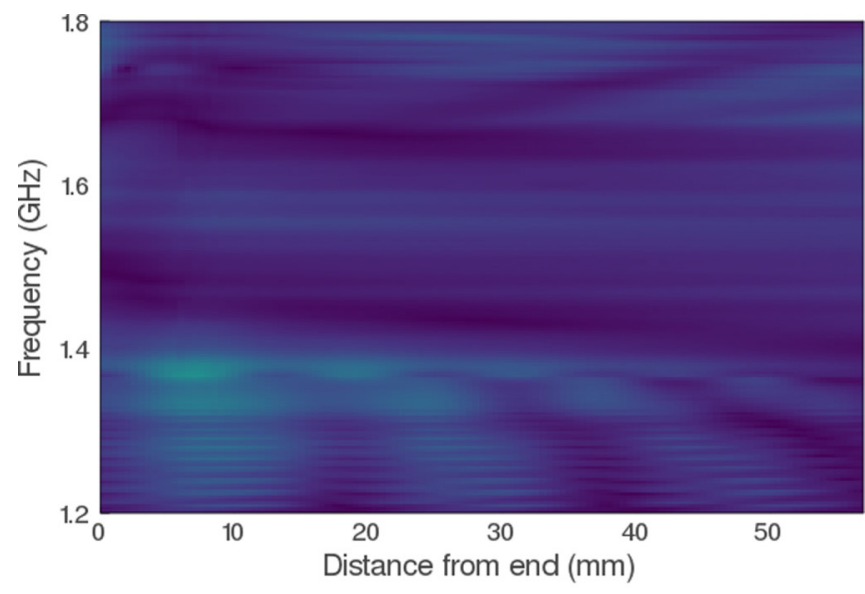

(a)

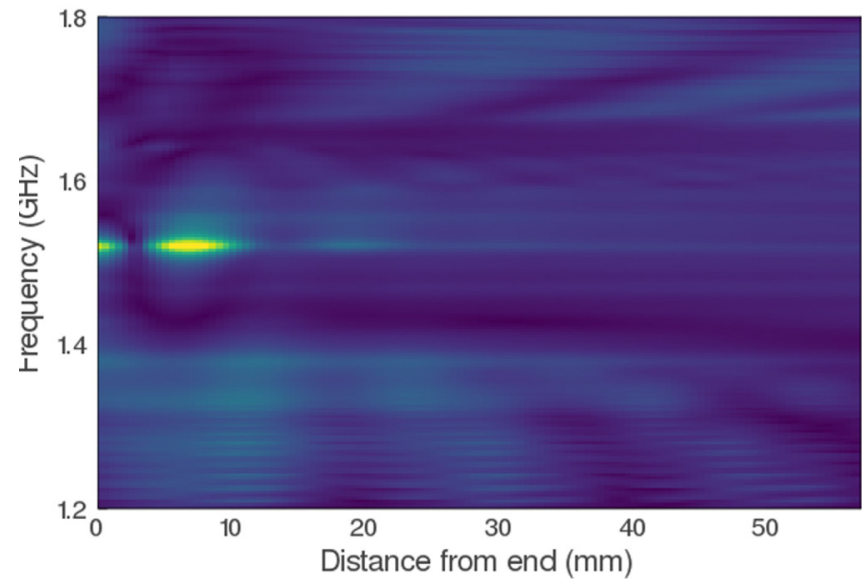

(b)

FIG. 6. Termination defect modes. (a) The magnitude of the transmission between the exciting and probing antennas as a function of frequency and distance along a $60 \mathrm{~mm}$ section at one end of a chain with $k_{B}>k_{A}$. Collective modes of the entire chain are evident as oscillations in the transmission for frequencies above and below the band gap (between 1.38 and $1.65 \mathrm{GHz}$ ). No modes are evident within the band gap. (b) The same as (a) but with $k_{A}>k_{B}$. In this case a localized mode confined to the end of the chain within the band gap occurs at $1.52 \mathrm{GHz}$. In both plots light green is used to show areas of high transmission while dark blue shows areas of low transmission. For both a change from maximum to minimum represents a $100 \%$ change in magnitude.

bulk of the chain will not be excited by an antenna placed at one end. Instead we sweep the two antennas in tandem along opposite sides of the chain, see bottom of Fig. 5, while recording the transmission between them as a function of distance and frequency.

In Fig. 7(a) we show the transmission data for the chain designed to support a symmetric boundary mode, see Fig. 4 for details. Note that we are only presenting data over a relatively narrow range of frequencies and distances as compared to Fig. 6 so as to identify more clearly the boundary mode, which is evident as a localized oscillation at a frequency of $1.42 \mathrm{GHz}$, within the band gap of both subchains. The data presented here are markedly different to those shown in Fig. 6, and this is a result of the different measurement setups. Firstly, 


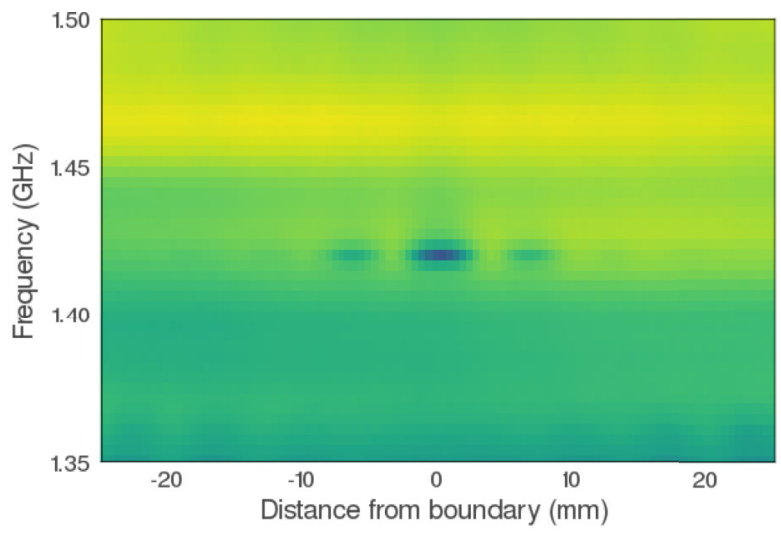

(a)

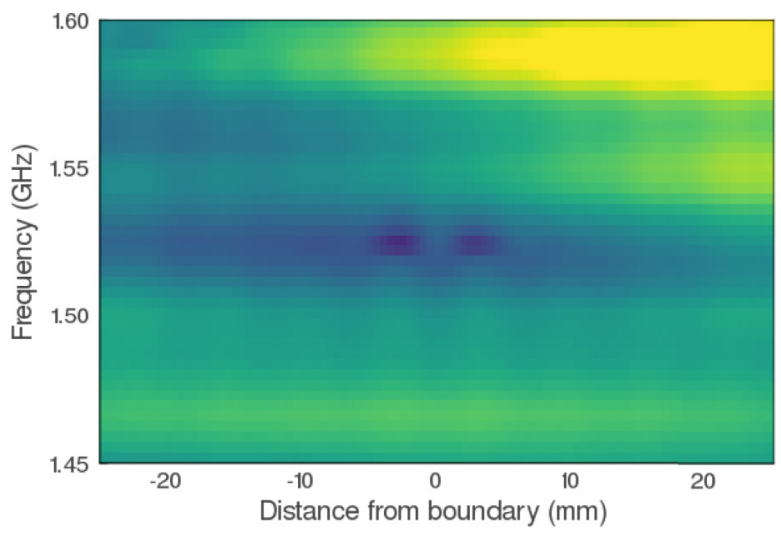

(b)

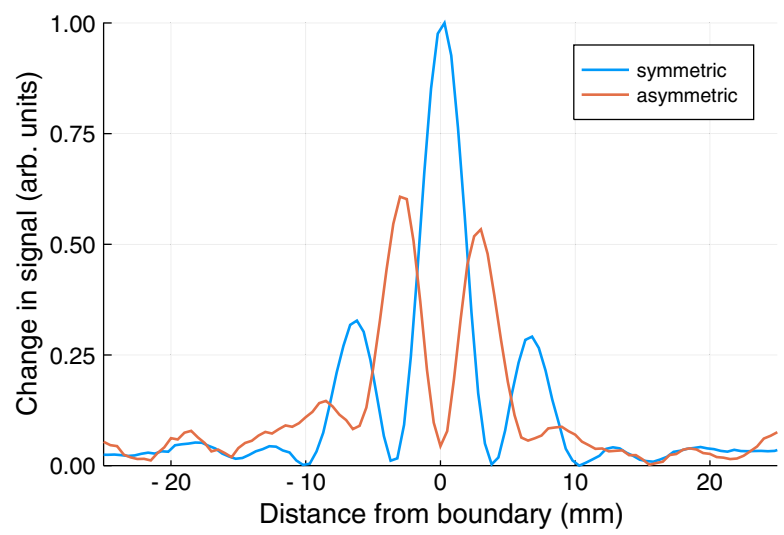

(c)

FIG. 7. Boundary defect modes. (a) The magnitude of the transmission between the exciting and probing antennas as a function of frequency and distance along a chain that supports a symmetric localized mode at the boundary between subchains with $k_{A}>k_{B}$ and $k_{A}<k_{B}$. The mode is observed as a localized oscillation in the transmission at the boundary at a frequency of $1.42 \mathrm{GHz}$. (b) The same as (a) but for a chain that supports an asymmetric localized mode. In this case the localized mode occurs at a frequency of $1.526 \mathrm{GHz}$. In both plots, light green is used to show areas of high transmission while dark blue shows areas of low transmission. For (a), a change from maximum to minimum represents a $100 \%$ change in magnitude while for (b) the same color shift is only a $62 \%$ change in magnitude. (c) The absolute value of the change in the transmission for the two modes (symmetric at $1.42 \mathrm{GHz}$, asymmetric at $1.526 \mathrm{GHz}$ ) demonstrating the different symmetries. [Note that background slopes in the data for (c) have been removed for clarity.] there is significant direct transmission between the antennas giving a much larger background transmission. Secondly, as opposed to observing maxima in the transmission as evidence of a mode, here we see minima. This is a result of energy that would have been transmitted directly between the antennas now being coupled into the mode and thereby lost to absorption. Thirdly, the measurement effectively rectifies the signal as a result of the antennas being swept in tandem.

While the observation of the predicted symmetric mode is pleasing, we have to note a disparity between our results and the predictions of the SSH model. Our SSH model predicts that the symmetric boundary mode should appear at the midpoint of the band gap, whereas in our experiment we observe it at a reduced frequency, closer to the lower energy band edge. In the simple SSH model all resonators are treated as identical with only the coupling strength between them altering. However, our system is rather more complex, with the resonance frequency of an oscillator being determined not only by its structure but also by its environment. In all other cases presented in this work each resonator has one neighboring resonator in close proximity (one spacer away) and one that is more distant (three spacers away). However, in the present case the resonator at the boundary between the subchains has no neighboring resonator nearby with both adjacent resonators being three spacers away. This results in the mode occurring at a lower frequency. However, we do not feel this harms the validity of our metamaterial comparison. Gap states in true polyacetylene, and other soliton supporting structures, also appear asymmetrically within their associated band gaps [18,22-24].

In Fig. 7(b) we present similar data but for the chain that was designed to support an asymmetric boundary mode. Once again, a localized oscillation is observed at the boundary between the subchains and, as expected, the mode occurs at a frequency of $1.52 \mathrm{GHz}$ (in the middle of the band gap). To confirm the difference in symmetry of the boundary modes supported by our two chains, in Fig. 7(c) we present the normalized absolute value of the change in transmission as a function of distance from the boundary for both cases. Note that background slopes in the data have been removed for clarity. Comparison between the traces in Fig. 7(c) and the eigenvectors in Fig. 2 show that these two features are well matched in form with those predicted by the SSH model. That is, in the symmetric boundary case the fields are concentrated around the central resonator at the boundary, while in the asymmetric case the central resonator is at a null.

\section{CONCLUSIONS}

We have directly observed two classes of defect modes in molecular aggregate-like metamaterial chains at microwave frequencies - 'edge modes' localized at the ends of the chains and 'boundary modes' localized at the boundary between subchains of differing design. We have taken advantage of the $\mathrm{cm}$ scale structures to probe the modes at a resolution deep within their near fields and have compared our results to predictions based on a simple Su, Schrieffer, and Heeger (SSH) model, showing qualitative agreement. Our results further demonstrate the capability of metamaterial analogs to mimic molecular aggregates, allowing the physics that un- 
derpins their behaviors to be explored in ways not possible in molecular systems. It would be interesting to see whether dynamic effects could be included in analog systems such as these, for example noise, the presence of which may well be important to real molecular systems [25].

\section{ACKNOWLEDGMENTS}

The authors would like to acknowledge input from Sathya Sai Seetharaman in the design of the metamolecule, and Nick
Cole and Peter Savage for fabricating essential equipment. We acknowledge financial support from the Engineering and Physical Sciences Research Council (EPSRC) of the United Kingdom, via the EPSRC Centre for Doctoral Training in Metamaterials (Grant No. EP/L015331/1). W.L.B. acknowledges the support of the European Research Council through project Photmat (ERC-2016-AdG-742222 [26]). I.R.H. acknowledges support from the EPSRC and QinetiQ Ltd. via the TEAM-A prosperity partnership (Grant No. $\mathrm{EP} / \mathrm{R} 004781 / 1)[27]$.
[1] M. Kasha, H. R. Rawls, and M. Hraf el Bayoumi, Pure Appl. Chem. 11, 371 (1965).

[2] F. C. Spano and C. Silva, Annu. Rev. Phys. Chem. 65, 477 (2014).

[3] T. Brixner, R. Hildner, J. Köhler, C. Lambert, and F. Würthner, Adv. Energy Mater. 7, 1700236 (2017).

[4] M. Gao and B. Z. Tang, ACS Sensors 2, 1382 (2017).

[5] C. J. Murphy, Anal. Chem. 74, 520 A (2002).

[6] G. McDermott, S. M. Prince, A. A. Freer, A. M. Hawthornethwaite-Lawless, M. Z. Papiz, R. J. Cogdell, and N. W. Isaacs, Nature (London) 374, 517 (1995).

[7] P. Qian, C. A. Siebert, P. Wang, D. P. Canniffe, and C. N. Hunter, Nature (London) 556, 203 (2018).

[8] J. Moll, S. Daehne, J. R. Durrant, and D. A. Wiersma, J. Phys. Chem. 102, 6362 (1995).

[9] T. Stangl, P. Wilhelm, K. Remmerssen, S. Höger, J. Vogelsang, and J. M. Lupton, Proc. Natl. Acad. Sci. 112, E5560 (2015).

[10] N. J. Hestand and F. C. Spano, Chem. Rev. 118, 7069 (2018).

[11] M. Baraclough, S. S. Seetharaman, I. R. Hooper, and W. L. Barnes, ACS Photonics 6, 3003 (2019).

[12] D. R. Smith, J. B. Pendry, and M. C. K. Wiltshire, Science 305, 788 (2004).

[13] J. Knoester, Proceedings of the International School of Physics "Enrico Fermi" Course Clix, (IOS Press, Amsterdam, 2002), pp. 149-186.

[14] K. Rustomji, M. Dubois, B. Kuhlmey, C. M. de Sterke,
S. Enoch, R. Abdeddaim, and J. Wenger, Phys. Rev. X 9, 011041 (2019).

[15] W. P. Su, J. R. Schrieffer, and A. J. Heeger, Phys. Rev. Lett. 42, 1698 (1979).

[16] N. Batra and G. Sheet, Resonance 25, 765 (2020)

[17] E. J. Meier, F. A. An, and B. Gadway, Nat. Commun. 7, 13986 (2016).

[18] S.-R. E. Yang, Nanomaterials 9, 885 (2019).

[19] J. B. Pendry, A. J. Holden, D. J. Robbins, and W. J. Stewart, IEEE Trans. Microwave Theory Tech. 47, 2075 (1999).

[20] M. Baraclough, I. R. Hooper, and W. L. Barnes, Phys. Rev. B 98, 085146 (2018).

[21] S. S. Seetharaman, C. G. King, I. R. Hooper, and W. L. Barnes, Phys. Rev. B 96, 085426 (2017).

[22] W. P. Su, J. R. Schrieffer, and A. J. Heeger, Phys. Rev. B 22 , 2099 (1980).

[23] A. J. Heeger, S. Kivelson, J. R. Schrieffer, and W. P. Su, Rev. Mod. Phys. 60, 781 (1988).

[24] A. M. Asaduzzaman, K. Schmidt-D'Aloisio, Y. Dong, and M. Springborg, Phys. Chem. Chem. Phys. 7, 2714 (2005).

[25] S. K. Saikin, M. A. Shakirov, C. Kreisbeck, U. Peskin, Y. N. Proshin, and A. Aspuru-Guzik, J. Phys. Chem. C 121, 24994 (2017).

[26] www.photmat.eu.

[27] M. Baraclough, I. Hooper, and W. Barnes, dataset for "Direct observation of defect modes in molecular aggregate analogues", University of Exeter, doi: 10.24378/exe.2843. 